\title{
Seniors Road Safety Enhancement through Perceptual- Motor Competences Training Program Impact upon the Performance on the Useful Field of Vision (UFOV)
}

\author{
Rui Matos • Isabel Varregoso • Ana Comprido • \\ Luís Coelho • Pedro Morouço • Nuno Amaro • \\ Marisa Barroso
}

Published online: 23 October 2012

(C) Springer Science+Business Media New York 2012

\begin{abstract}
The purpose of this study was to verify the efficacy of a PerceptualMotor Competences Training Program upon the performance on UFOV of an experimental active group of elderly drivers. Program tasks were conceived to force subjects to divide their attention for central and peripheral stimuli, using a visual strategy of gazing at an anchor-point and, from there, detect important clues on peripheral visual field. For this purpose, almost all tasks were performed on groups of two, being one responsible for testing the other and switching functions from time to time (for instance, dropping one of two juggling handkerchiefs hold apart, one in each tester hand, with the partner having to detect which was dropped and react as quick as possible, catching it before it felt to the floor). We found a significant improvement in the capacity of dividing attention for central and peripheral stimuli under a visual environment full of distractors (selective attention UFOV test) following the Program, as well as a significant reduction on the category of risk (component based upon the three UFOV subtests results), which evidence very clearly the benefits of this training program on the elderly experimental group and its potential to reduce their risk of being involved in car accidents, on their fault, due to perceptual reasons.
\end{abstract}

Keywords Car driving · Peripheral vision · Elderly · Training

R. Matos $\cdot$ I. Varregoso $\cdot$ A. Comprido $・$ L. Coelho $\cdot$ P. Morouço $・$ N. Amaro $・$ M. Barroso School of Education and Social Sciences, Polytechnic Institute of Leiria, Leiria, Portugal

R. Matos · I. Varregoso $\cdot$ A. Comprido $\cdot$ L. Coelho $\cdot$ P. Morouço $\cdot$ N. Amaro $・$ M. Barroso Research Center for Human Movement Science, Polytechnic Institute of Leiria, Leiria, Portugal

R. Matos $(\bowtie)$

Escola Superior de Educação e Ciências Sociais (Leiria), Campus 1 Rua Dr. João Soares Apartado 4045, 2411-901 Leiria, Portugal

e-mail: rui.matos@ipleiria.pt 


\section{Introduction}

Car driving can be seen as a perceptual-motor task which requires a continuous surveillance not to make mistakes that can result into accidents. According to Brown (1990), it's probable that drivers, every day, exhibit a large amount of errors correction behaviour, with different amounts of success. Therefore, it's essential to maintain a high state of attention to face unpredictable events, so that this driving gets performed in a safe way, through shorter or longer periods and, often, undertaken on monotonous conditions.

Since world population is aging, it's important to study how elderly driving capacity declines and if it is possible to do something to avoid or, at least, diminishes its intensity.

Sekuler et al. (2000) state that driving efficiency reduction in elderly is enhanced when conditions require attention division for central and peripheral tasks. They also say that previous research showed that useful field of vision (UFOV) is a trustable tool to predict driving ability and that it seems to decline with aging. If it happens to be a problem on peripheral vision, due to structural reasons or to a cognitive or foveal overload, namely by cell phone use, talking to passengers in the vehicle, etc., drivers may not be able to react to some hazard coming from far left or right (horizontal peripheral vision) or to detect traffic lights that are placed over a cross (vertical peripheral reason). They can, also, have to make sudden movements when performing a bend or drive too close to precedent car (tailgating) or cars that are stopped on street edges.

Sekuler et al. (2000) concluded that UFOV deterioration begins early (at the age of twenty or even before). This deterioration is more sharply conceptualized as a reduction upon the efficiency with which people can extract information from a complex scenario (e.g., multiple lanes, many vehicles) and not, mainly, as a narrowing per se of UFOV (as pointed out by Langham et al. 2002, stressing the importance of attentional fails on the occurrence of several accidents). Also Rumar (1990) pointed the finger to failures on cognitive expectancies or difficulties on dealing with perceptual boundaries.

Wood and Troutbeck (1995) found a correlation between UFOV and driving performance in a closed circuit. UFOV was, also, found to be correlated with driving simulator performance Rizzo et al. (1997). Therefore, UFOV test seems to capture higher order visual processes that moderately correlate with performance in a variety of cognitive tests. It also seems to provide unique information about the risk involved in car driving. In fact, Ball et al. (2002), after having made a revision of studies, found that subjects performance in UFOV seems to be a stronger accident occurrence predictor then visual acuity, mental status tests or precocious dementia diagnostic.

Ball et al. (1993) remember that peripheral vision seems to play a fundamental role on driving, since a severe visual field lost in both eyes will double the risk of having an accident; at the same time, they affirm that is growing exponentially the number of studies that show that the bigger the UFOV deficiency, the bigger the risk of having problems on car driving, including a raised probability of accidents occurrence. Roenker et al. (2003) highlight that older drivers are slower on making driving decisions but, if they have the time, the quality of those decisions is not worse. The problem is that those decisions, that we have to take when facing unexpected events 
on driving and that require quick answers, are the ones that have been associated to an augmented accident risk in those older drivers. This situation, nevertheless, can be, at least partially, reverted, through a information processing speed training, as shown by these authors with elderly, where UFOV increased with training, consequently diminishing hazard manoeuvres on driving.

Isler et al. (1997) studied the capacity of different age drivers at several driving relevant visual functions, with horizontal peripheral vision test showing that people over 60 obtained the worse results and with the situation becoming even worse with age advance. Lee et al. (2003), studying visual attention through reaction times to a sequence of visual stimuli, during the main driving task, also showed that it declined with age. Maltz and Shinar (1999), showed that aging can affect visual information processing efficacy and recommended that older drivers should train visual search. Marmeleira et al. (2009) also demonstrated that abilities associated with driving performance in older adults could get better through a 12-weeks ( 3 sessions a week) perceptive, cognitive and physical exercise program. In the same line, Marmeleira et al. (2011), studying the effects of a specific exercise program upon some features of on-the-road driving, concluded that exercise can enhance speed of behavior in older drivers and should, therefore, be promoted.

As a complementary perspective, literature gives us several examples of better visual information processing of expert sport players, as compared to rookies (e.g., Helsen and Pauwels 1993; Huys and Beek 2002; Kioumourtzoglou et al. 1998, better peripheral vision of people involved in sport, compared to those who are not (e.g., Cockerill 1981; Davids 1984; Matos 2008), better reaction times (e.g., Ando et al. 2001) and, probably, some advantage in certain features of driving tasks (e.g., Kane et al. 1999; Hancock et al. 2002; Matos 2008).

Matos (2008) showed that it was possible to train young drivers to enhance perceptual-motors abilities, namely the capacity to divide attention through central and peripheral stimuli, associated to a better use of peripheral vision.

In this study, it was our intention to verify the efficacy of a Perceptual-Motor Competences Training Program upon the performance on UFOV of an experimental active group of old drivers from Leiria.

\section{Methods}

\section{Participants}

Twelve subjects, being 5 males (Mean age 66,69 $\pm 5,07$ ) and 7 females (Mean age $71,96 \pm 4,49$ ), participated. They were divided into 2 groups (Experimental and Control), as follows (Table 1):

Table 1 Participants caracterization

\begin{tabular}{|c|c|c|}
\hline Group & Total & Age $(\mathrm{M} \pm \mathrm{DP})$ \\
\hline Experimental & $6(2 \widehat{\jmath}, 4$ ㅇ $)$ & $69,2 \pm 6,53$ \\
\hline Control & $6(3 \widehat{\jmath}, 3$ ㅇ $)$ & $68,6 \pm 4,19$ \\
\hline Total & $12(5 \hat{\jmath}, 7$ ○) & $68,9 \pm 5,49$ \\
\hline
\end{tabular}


These subjects integrate an autarchic Physical Education Program, where they practice twice a week (one session of hydrogymnastics and another of gymnastics). They all signed an informed written consent, after presentation of the study, where they stated they agreed on being UFOV tested and, if making part of the experimental group, participating on the Training Program.

\section{Procedure and Measures}

Procedure took in account Declaration of Helsinki and was approved by Research Center for Human Movement Science ethical committee. We carried out, with the experimental group, 10 sessions (twice a week, 5 weeks in a row) of 45 min each, in a gym. In a prior study (Matos 2008), despite it was conducted with youngsters, we stated that it was important to conduct these exercises in group and not individually, mainly because the interaction, itself, would proportionate situations that, performed alone, would be more difficult to happen. Also, some exercises needed several persons to be performed and, above all, exercises would be much more funny and enjoyable performed in group! Exercises, adapted from Matos (2008), were chosen aiming to develop, mainly, the capacity to divide attention through central and peripheral stimuli, associated to a better use of peripheral vision. The main goal was to force subjects to pay simultaneous and divided attention to more than a spot on his visual field, using an anchor-point, that is, gazing at a point equidistant from two sources of information (for instance, two juggling handkerchiefs hold apart, with only one meant to be dropped at a time) and, from there, detect important clues on peripheral visual field - advanced signs of the beginning of one of the handkerchiefs dropping - which to react.

The ones we quickly describe below are those we consider more effective and representative of the Program tasks. In Sardine game task (Fig. 1), two subjects stand up facing each other. One of them holds his hands with palms up and the other puts his over partner hands, palms facing down, without contacting partner ones (to prevent tactile information). The purpose is that the one who has his hands below makes a quick hands move, so that he manages to hit, with either of them, one of his partner hands back, who tries to avoid it. To make the task harder and more demanding, after some tries hands were to be held wider and wider apart, so that information became more eccentric in the visual field. In Handkerchiefs fall and catch task (Fig. 2), one of the subjects drops one of two juggling handkerchiefs he

Fig. 1 Sardine game task

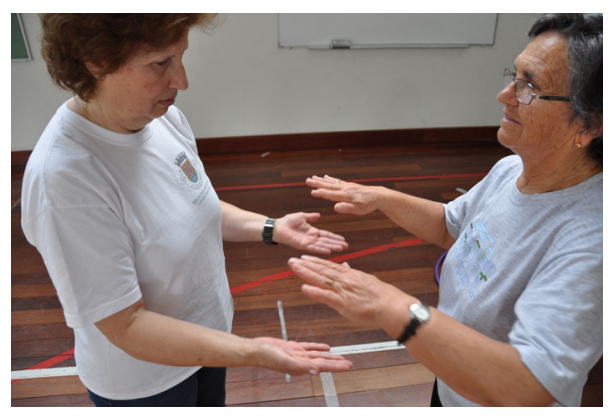


Fig. 2 Handkerchiefs fall and catch task

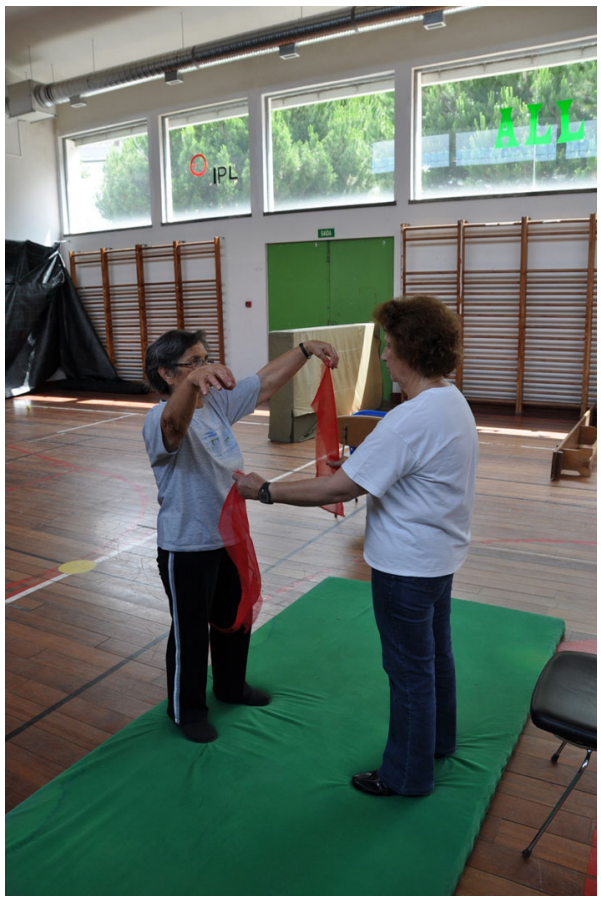

holds apart, one in each hand, with the partner having to detect which was dropped and react as quick as possible, catching it before it felt to the floor. The strategy described to make the first task more demanding was also used in this one. Another exercise we used was Goalkeeping task (Fig.3). One of the subjects acting as a goalkeeper, trying to defend a ball thrown by one of his colleagues of experience. These are coupled organized, holding, each of them, a ball. Goalkeeper never knows which of them will throw the ball (despite they all have a ball, only one in each pair throws it, following a previous and secret combination between them. The one who doesn't throw the ball simulates he will, making an acceleration arm movement at the same time of his partner but without releasing it). Despite the exercise is mainly intended to make the goalkeeper (every subject assumes this function on his time) develop his/her peripheral vision/attention division, throwers also develop it, since

Fig. 3 Goalkeeping task

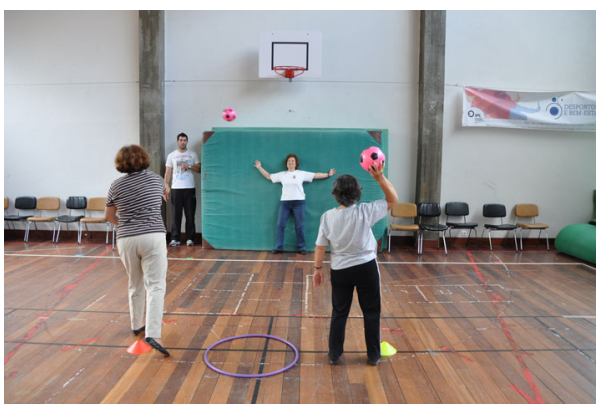


they have to, peripherally, visually control his partner performance, so that their movements are as simultaneous as possible, to force the goalkeeper to be unsure till the last moment. One fourth exercise was Hit the ball rolling from behind task (Fig. 4). One subject, holding a tennis racket, with all his colleagues behind him, tries to hit the balls they put to roll without looking back over the shoulders. These balls (one at a time) may appear from his right or from his left side. After some strokes, another subject assumes his position, allowing everybody to try it. One last example is Press the pushed stick task (Fig. 5). On pairs, the subject seated in front tries to press, stopping it, as soon as possible, a stick pushed from behind by his partner. Similarly to tasks 1 and 2, it's important that front subjects' hands do not contact sticks in preparatory phase, so that clues remain strictly visual.

The measures we took were processing speed, divided attention and divided and selective attention (UFOV results), as well as category of risk, a component based upon the three UFOV subtests results. UFOV is a computer-administered and computerscored test of functional vision and visual attention, which can be predictive of ability to perform many everyday activities, such as driving a vehicle. It is specially recommended for people whose age is 55 years old or older. Processing speed, the first measure we mentioned, was measured by UFOV first subtest, which asked subjects to detect if a symbol that was briefly presented was a car or a truck (which differ just slightly). Besides processing speed, subjects central vision was also tested. The second UFOV subtest, more complex, forced subjects to focus their gaze at the centre of computer monitor, so that they would be able to make the distinction we mentioned about UFOV subtest 1. At the same time, using peripheral vision, subjects had to detect the position (one among pre-defined eight) of another symbol briefly exposed, eliciting, this way, the use of divided attention. The last UFOV subtest was quite similar to the previous one with a unique difference: the symbol that emerged in one of eight possible eccentric positions was, somehow, camouflaged by a series of white triangles, making appeal to the third measure we took and analysed, divided and selective attention.

These three measures were all registered in milliseconds, representing the smallest amount of time needed to detect the stimuli (central and peripheral) we described. Being 17 milliseconds the time of a refreshing monitor cycle, that is, therefore, the best (minimum) time a subject can obtain in any of the three subtests. The other measure, category of risk, was automatically computer-scored, attending to three UFOV subtests results combination, ranging from 1 (very low risk) to 5 (high risk), representing 2 low risk, 3 low to moderate risk and 4 moderate to high risk.

Fig. 4 Hit the ball rolling from behind task

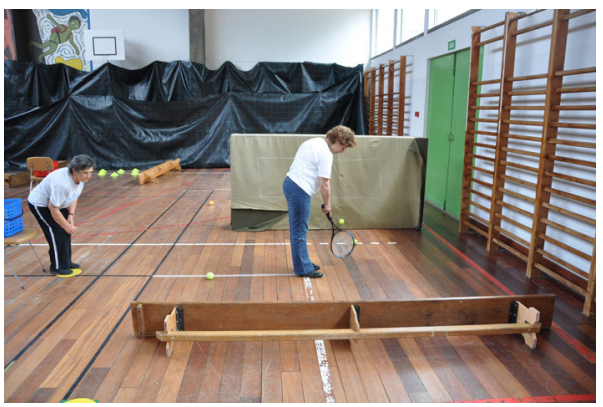


Fig. 5 Press the pushed stick task

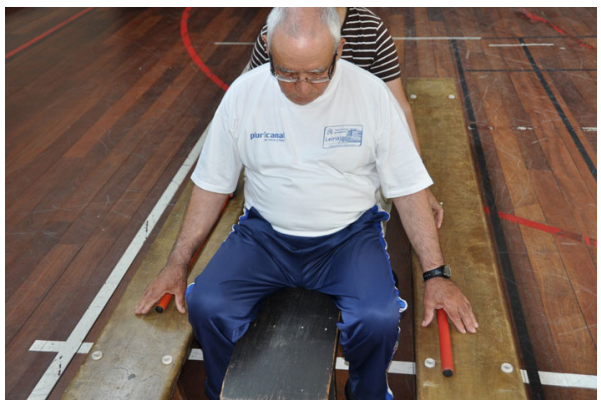

Data were analysed through a statistical analysis program - Statistical Package for the Social Sciences (SPSS), version 19.0. Non-parametric statistics was used, namely the Wilcoxon test (comparing results of each of the two groups before and after the training program), and the Mann-Whitney $U$ test (comparing results of experimental and control groups before and after the training program), setting the level of significance at $\mathrm{p} \leq 0.05$.

\section{Results}

As can be seen in Table 2, and contrary to the control group, the results of the experimental group showed, after the Perceptual-Motor Training Program, a statistically significant improvement upon selective attention $(Z=-1,992, p=.046$, Wilcoxon test) and category of risk, a global-factor component $(Z=-2,070, p=.038$, Wilcoxon test), on UFOV test. Processing speed and divided attention of experimental group subjects also improved but gains did not reach statistical significance.

Results also show that experimental and control group, though equivalent before program application, became significantly different (experimental group better) on the same parameters (selective attention and category of risk) after it $(Z=-2,286$, $p=.022$, and $Z=-2,514, p=.012$, respectively, Mann-Whitney $U$ test), on UFOV.

Table 2 UFOV results before and after the training program $(\mathrm{M} \pm \mathrm{SD})$

$\left.\begin{array}{llcc}\hline \text { Group } & & \text { Initial } & \text { After Program } \\ \hline \text { Experimental } & & & \\ & \text { Category of risk } & 2,67( \pm 0,816) & 1,50( \pm 0,548) * \\ & \text { Processing speed } & 43,33( \pm 44,374) & 20,65( \pm 6,786) \\ & \text { Divided attention } & 171,17( \pm 72,904) & 101,10( \pm 96,948) \\ & \text { Selective attention } & 356,33( \pm 96,432) & 251,27( \pm 87,688) * \\ \hline \text { Control } & & & \\ & \text { Category of risk } & 3,33( \pm 1,281) & 3,17( \pm 1,169)\end{array}\right]$

$* p \leq .05 * * p \leq .01$ 


\section{Discussion and Conclusions}

Though the reduced $n$ of our sample (several elements of our initial sample couldn't manage, despite initial compromise, to attend the sessions of the program), results seem pretty conclusive. In fact, the significant improvement in the capacity of dividing attention for central and peripheral stimuli under a visual environment full of distractors (selective attention), makes us very confident of Program' tasks potential to enhance these perceptual features. Also, and not least important, the results on the global-factor component represented by category of risk, which derives from combination of the three sub-tests of UFOV, evidence very clearly the benefits of this training program on seniors experimental group.

Our results come in line with previous findings and authors recommendations (Roenker et al. 2003; Maltz and Shinar 1999; Marmeleira et al. 2009, 2011), who showed that it was possible to train elderly and, consequently, enhance their visual attention and other perceptual features, diminishing, thus, hazard manoeuvres when driving.

We think that our results show, in fact, that it is possible to enhance elderly perceptual capacities, namely the ability to divide attention for central and peripheral stimuli. Our study also shows the effectiveness of an off-road training program on UFOV and, according to previous studies that highlight the correlation between UFOV results and road safety (Ball et al. 1993, 2002; Rizzo et al. 1997; Roenker et al. 2003; Wood and Troutbeck 1995), we can speculate that this training program may diminish seniors risk of being involved in accidents on their fault.

Acknowledgments We would like to thank Maria Correia, Luís Neves and Tiago Santos for their contribution to this paper, namely on their action of recruiting subjects to the study, UFOV testing and Program Training conception and implementation.

\section{References}

Ando, S., Kida, N., \& Oda, S. (2001). Central and peripheral visual reaction time of soccer players and nonathletes. Perceptual and Motor Skills, 92(3 Pt 1), 786-794.

Ball, K., Owsley, C., Sloane, M. E., Roenker, D. L., \& Bruni, J. R. (1993). Visual attention problems as a predictor of vehicle crashes in older drivers. Investigative Ophthalmology \& Visual Science, 34(11), 3110-3123.

Ball, K., Wadley, V., \& Edwards, J. (2002). Advances in technology used to assess and retrain older drivers. Gerontechnology, 1(4), 251-261.

Brown, I. D. (1990). Drivers' margins of safety considered as a focus for research on error. Ergonomics, 33 (10-11), 1307-1314.

Cockerill, I. (1981). Peripheral vision and hockey. In I. Cockerill \& W. Gillivary (Eds.), Vision and Sport (pp. 54-63). Cheltenham: Stanley Thornes Publishers Ltd.

Davids, K. (1984). The Role of Peripheral Vision in Ball Games: Some Theoretical and Practical Notions. Physical Education Review, 7(1), 26-40.

Hancock, P. A., Kane, M., Scallen, S., \& Albinson, C. (2002). Effects of gender and athletic participation on driving capability. International Journal of Occupational Safety and Ergonomics, 8(2), 281-292.

Helsen, W., \& Pauwels, J. M. (1993). The relationship between expertise and visual information processing in sport. In J. L. Starkes \& F. Allard (Eds.), Cognitive issues in motor expertise (pp. 109-134). Amsterdam: North-Holland.

Huys, R., \& Beek, P. J. (2002). The coupling between point-of-gaze and ball movements in three-ball cascade juggling: the effects of expertise, pattern and tempo. Journal of Sports Sciences, 20(3), 171-186. 
Isler, R. B., Parsonson, B. S., \& Hansson, G. J. (1997). Age related effects of restricted head movements on the useful field of view of drivers. Accident Analysis and Prevention, 29(6), 793-801.

Kane, M., Pearce, K., Hancock, P., Scallen, S., \& Heniff, C. (1999). Investigating differences in driver accident involvement: the influence of perceptual motor competence, competitive athletics, and gender. Minneapolis: Tucker Center for Research on Girls and Women in Sport, \& University of Minnesota.

Kioumourtzoglou, E., Kourtessis, T., Michalopoulou, M., \& Derri, V. (1998). Differences in several perceptual abilities between experts and novices in basketball, volleyball and water-polo. Perceptual and Motor Skills, 86(3 Pt 1), 899-912.

Langham, M., Hole, G., Edwards, J., \& O’Neil, C. (2002). An analyses of "looked but failed to see" accidents involving parked police vehicles. Ergonomics, 45(3), 167-185.

Lee, H. C., Lee, A. H., \& Cameron, D. (2003). Validation of a driving simulator by measuring the visual attention skill of older adult drivers. American Journal of Occupational Therapy, 57(3), 324-328.

Maltz, M., \& Shinar, D. (1999). Eye movements of younger and older adults. Human Factors, 41(1), 15-25.

Marmeleira, J. F., Godinho, M. B., \& Fernandes, O. M. (2009). The effects of an exercise program on several abilities associated with driving performance in older adults. Accident Analysis and Prevention, 41(1), 90-97.

Marmeleira, J., Melo, F., Tlemcani, M., \& Godinho, M. (2011). Exercise can improve speed of behavior in older drivers. Journal of Aging and Physical Activity, 19(1), 48-61.

Matos, R. (2008). Campo Visual Útil, Divisão da Atenção e Velocidade de Reacção Periférica - treinabilidade e transfer da prática desportiva para tarefas de condução automóvel. Doctoral Thesis (unpublished). Cruz Quebrada, Lisboa: Faculdade de Motricidade Humana.

Rizzo, M., Reinach, S., McGehee, D., \& Dawson, J. (1997). Simulated car crashes and crash predictors in drivers with Alzheimer disease. Archives of Neurology, 54(5), 545-551.

Roenker, D., Cissell, G., Ball, K., Wadley, V., \& Edwards, J. (2003). Speed-of-processing and driving simulator training result in improved driving performance. Human Factors, 45(2), 218-233.

Rumar, K. (1990). The basic driver error: late detection. Ergonomics, 33(10-11), 1281-1290.

Sekuler, A. B., Bennett, P. J., \& Mamelak, M. (2000). Effects of aging on the useful field of view. Experimental Aging Research, 26(2), 103-120.

Wood, J. M., \& Troutbeck, R. (1995). Elderly drivers and simulated visual impairment. Optometry and Vision Science, 72(2), 115-124.

Rui Matos $\mathrm{PhD}$ in Human Movement Sciences

Professor at Polytechnic Institute of Leiria

Having finished my $\mathrm{PhD}$ in 2008, I continued to investigate the role of peripheral vision on motor performance.

Doing that, I have found interesting connections between peripheral vision use and efficiency at different domains of activity, such as car driving, sport and even cinema (my paper "Peripheral Vision and screen best place for subtitles location" won the contest for best communication on Avanca Cinema, 2010, among almost 140 international papers coming from 20 different countries).

This paper is the result of an appliance to a Polytechnic Institute of Leiria Investigation Found.

Isabel Varregoso has a long career as academic in the Polytechnic Institute of Leiria. Recently has engaged in research activities regarding the Pedagogy of Sport and Physical Activity and Ageing. Areas of teaching expertise are didactics; active aging; lifelong learning; dance and dance for different populations; and motor activities for elderly.

Ana Comprido is an Assistant Professor at the High School of Education and Social Sciences of Polytechnic Institute of Leiria, waiting for doctoral thesis defence in the field of Physical Activity for Elderly. Areas of teaching activity are active aging; and motor activities for elderly. She has a career in Physical Activity with Older Populations, since 2002.

Luís Coelho is a Lecturer at High School of Education and Social Sciences of Polytechnic Institute of Leiria. Areas of teaching expertise are Motor Development; and Motor Control and Learning. His doctoral thesis focused on the analysis of students and teachers self-determined motivations towards Physical Education. He's now the Sports and Well-Being course coordinator. 
Pedro Morouço has a PhD in Sport Sciences and a strong research profile, with a major focus in Human Movement Analysis. His research and publication agendas have focused on Biophysical Evaluation, namely the constraints induced by ageing. He's now the Executive Director of the Research Centre for Human Movement Sciences.

Nuno Amaro is an Assistant Professor at the High School of Education and Social Sciences of Polytechnic Institute of Leiria and an effective member of the Research Centre for Human Movement Sciences. Currently he's undertaking his doctoral degree in Sport Sciences at the University of Beira Interior.

Marisa Barroso is an Assistant Professor at the High School of Education and Social Sciences of Polytechnic Institute of Leiria with activities in the field of Folk Dance for Elderly. Currently she's undertaking her doctoral degree in Sport Sciences at Madeira University in association with High School of Sport of Rio Maior - Polytechnic Institute of Santarém. 